\title{
Study of Buckling Behavior of Beam and Column
}

\author{
${ }^{1}$ Rekha M. Bhoi, ${ }^{2}$ Prof. L. G. Kalurkar \\ ${ }^{I}$ Department of Civil, PG Student, J.N.E.C. Engg College, Aurangabad, 431003, India \\ ${ }^{2}$ Department of Civil, Faculty of Technical education, J.N.E.C. Engg College, Aurangabad, 431003, India
}

\begin{abstract}
The objective of this work is to study the buckling behavior of beam and column and effect of buckling behavior of beam and column. These structural members are subjected to heavy loads and can experience failure due to buckling. The method includes the effects of flange and web interaction, residual stresses, and elastic and inelastic behavior.
\end{abstract}

Keywords: Beam, Column, Buckling.

\section{Introduction}

Buckling is that mode of failure when the structure experiences sudden failure when subjected to compressive stress. When a slender structure is loaded in compression, for small loads it deforms with hardly any noticeable change in the geometry and load carrying capacity. At the point of critical load value, the structure suddenly experiences a large deformation and may lose its stability to carry load. This stage is the buckling stage.

The critical buckling load for a column is given by the formula

Where,

$$
\text { Pcr }=\frac{\text { EI } \pi^{2}}{\text { Leff }^{2}}
$$

$$
\begin{aligned}
& \mathbf{P}_{\text {cr }}=\text { Critical buckling load } \\
& \text { EI = Flexural rigidity } \\
& \mathbf{L}_{\text {eff }}=\text { Effective length }
\end{aligned}
$$

This formula is also called as Euler Critical Buckling Load Formula. The structural instability of buckling can be categorized as

\section{Bifurcation buckling}

In Bifurcation buckling the deflection when subjected to compressive load, changes from one direction to a different one. The load at which bifurcation occurs is the Critical Buckling Load. The deflection path that occurs prior to the bifurcation is called as the Primary Path and that after bifurcation is called as secondary or post buckling path.

Local buckling of "I" shapes may be defined as a bifurcation phenomenon whereby a component plate or plates subjected to in-plane stresses may be in equilibrium in an original planar configuration or in a neighboring deflected configuration. This critical state can occur in the elastic or inelastic regions of material response depending on the yield stress level, the plate width-to-thickness ratios, and the plate boundary conditions. Performance and strength checks required for individual beams or columns, all modern steel specifications also require that the local buckling of a cross section be examined. This same general philosophy also applies in the case of a beam-column, i.e., local buckling must not precede the attainment of the crosssectional capacity of the member. An investigation of buckling behavior of steel beam-columns having an $\mathrm{H}$ shaped cross section were studied for their deform ability as well as ultimate strength. A constant axial thrust and monotonically increasing end moments were applied to the beam-columns. Numerical analysis demonstrated its capability to properly simulate the behavior of the tested beam-columns. On the basis of parametric study using the numerical analysis, important findings can be drawn. First, lateral torsional deflection is more distinguished for beam-columns with less axial force. Second, beam-columns having a small slenderness ratio do not lead to instability by lateral torsional buckling, indicating a stable plastic range. Third, deformability drastically decreases once the slenderness ratio exceeds a certain level. Available formulas which estimate the ultimate strength of beam-columns, in addition, are found to be effective. Structural members subjected to axial thrust and bending moment in the strong axis are classified as Beam-columns. The beamcolumn has two modes of failure. If the beam-column is stocky, it fails due to excessive bending in its strong axis. If it is slender, a buckling, called the lateral torsional buckling, occurs, and the beam-column fails by the combined effect of in-plane and lateral deformations. Beam-columns failing in this latter mode can further be divided into two groups in accordance with their slenderness. If the beam-column is very slender, an elastic lateral torsional buckling occurs, ultimately causing failure. If the beam-columns is relatively stocky, on the 
other hand, it buckles first but may sustain more load or deform further without losing its load carrying capacity. Structural engineers must accurately evaluate the strength of such beam-columns in designing structures. In plastic design or earthquake resistant design, the deformability of structural members is as important as their strength. In plastic design, this deformability is expressed in terms of the rotation capacity. Plastic hinges in beam-columns of a structural frame are required to maintain plastic moments until the frame for Extensive research, both experimental and theoretical, has been performed on the in-plane behavior of beam-columns, providing various useful equations to predict ultimate strength. Closed form solutions are also available for computing the elastic lateral torsional buckling load of beam-columns. Current research, therefore, focuses on beam-columns which buckle in the inelastic range and which possess the ability to carry external forces in the post-buckling range. One can also find works which were devoted to investigating the deformability of beamcolumns. An analytical procedure to predict the rotation capacity of beams that fail ultimately either by excessive lateral deformation or by local buckling and demonstrated the capability of his procedure to properly estimate the rotation capacity Local buckling of I shapes may be defined as a bifurcation phenomenon whereby a component plate or plates subjected to in-plane stresses may be in equilibrium in an original planar configuration or in a neighboring deflected configuration. This critical state can occur in the elastic or inelastic regions of material response depending on the yield stress level, the plate width-to-thickness ratios, and the plate boundary conditions. In the case of $\mathrm{W}$ shapes it has been shown that the complex interaction of web and flange plates also plays a significant role in the bifurcation behavior of the section. The general formulation for thinwalled members, described elsewhere, is used in the present study to develop a mathematical formulation whereby local buckling behavior may be investigated in depth for $\mathrm{W}$ shape members end-loaded under combined axial and flexural stresses. The effects of a general residual stress pattern as well as the effects of elastic and inelastic material response and the interaction effects of web and flange plates are included in the analysis. Buckling of the structures is dependent on various numbers of variables like geometry, material properties and the applied load. Stability theory, which had its beginning approximately 250 years ago, when Euler first calculated the load carrying capacity of an elastic column, has made its greatest strides forward during the last 50 years. In the 1930's, the failure of plates at loads considerably in excess of the critical load was investigated plates loaded in shear, and for axially compressed plates. This was followed in the early investigation of the collapse of axially compressed cylindrical shells at loads far below the critical load, and in general stability theory which explained the buckling behavior, and provided a firm theoretical foundation for the entire subject. Due to the stimulus produced by these as well as other pioneering studies, the past 50 years have witnessed far reaching advances in our understanding of buckling behavior. An effective section method is presented for analyzing the effects of local buckling on the overall modes of behavior of singly symmetric to beam-columns.

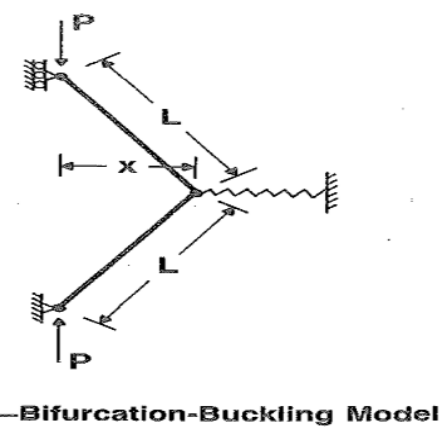

The most general type of member in a steel framework is a beam - column, which transfers the applied loads by a combination of bending and axial-compression actions. Beam-columns may act as if isolated, or they may be continuous members that form parts of a rigid frame. In the plastic method of design of planar rigid frameworks, the beam - columns are assumed to be subjected to axial loads and major axis moments only, with out-of-plane deformation prevented by lateral bracing. However, when the lateral bracing is insufficient, the beam-column will fail by inelastic lateral buckling at a lower load than that which causes plastic collapse. This inelastic buckling load is reduced below the elastic buckling load due to the significant effects of the yielding that occurs in these members of low to intermediate slenderness. While the inelastic lateral buckling of continuous beams has been studied by only a few researchers, even fewer investigations have been made of the inelastic lateral buckling of continuous beam-columns.

\section{Analytical Procedure}

In the analytical procedure, a constant load $\mathrm{P}$, such that $0<\mathrm{P}<\mathrm{Py}$, where Py is the yield load, is applied to a section. Local buckling under pure axial load is checked to ensure that the section does not buckle 
under the applied load. A bending moment is then applied to the section and gradually incremented until local buckling occurs under the combined loading. Because of the interaction of axial force, bending moment, and residual stresses, it is possible that partial or complete inelastic behavior results and an iterative technique is therefore required.

\section{Parametric Study}

As for a beam, a beam-column should be identified as to the desired strength and rotation capacity.

The categories usually used are:

1. Attainment of the plastic moment plus rotation capacity sufficient for redistribution of moments. These are called plastic design sections.

2. Attainment of plastic moment capacity only. These are called compact sections,

3. Attainment of yield moment only. These are called non-compact sections.

However as the flanges are concerned, it can be assumed that the local buckling of a beam-column section is not distinguishable from the local buckling of a beam section. This is only approximately true. Although it is reasonable to assume in both cases that the flange is acting under a uniform strain, there is some buckling interaction between web and flange. The web ofa beam will be subjected to a linear strain gradient as a consequence of the bending moment, but the web of a beam-column will see the effects of both the axial force and the moment. Nevertheless, in order to reduce the number of variables, it will be convenient to use the assumption that the flanges of beam-columns will be governed by the same rules as the flanges of beams. The interaction of the flange and web elements during local buckling is taken into account in the analytical procedure.

\section{Compact Beam-Columns.}

Local buckling interaction curves for various values and a fixed flange slenderness for compact beamcolumns. As the ratio $\mathrm{MCr} / \mathrm{Mp}$ increases from zero, each local buckling curve becomes tangential to the strength interaction line, $\mathrm{Mpc}=\mathrm{Mp}(\mathrm{l}-\mathrm{P} / \mathrm{Py})$, where $\mathrm{Mpc}$ is the reduced plastic moment in the presence of axial load. At a point of tangency, the mode of failure changes from web local buckling to flange local buckling. The values P/Py established in this way for the various curves represent optimum values, wherein a section capacity based on strength coincides with its local buckling capacity. The straight-line approximation to the optimum local buckling curve, results in conservative limitations, when values of P/Py are less than about 0.2. This is desirable, however, in order to avoid the sensitivity of a steep gradient in this region and to recognize the possibility of small axial loads that may occur in service but that are not taken into account in the analysis of the structure.

\section{Plastic Design Beam-Columns.}

Interaction relationships of $\mathrm{P} / \mathrm{Py}$ versus $\mathrm{Mcr} / \mathrm{Mp}$ for various values are shows graphically, for a fixed value of flange slenderness, corresponding to a plastic design section. The bilinear and linear dashed curves. The points of intersection of the interaction curves with the bilinear dashed curve represent optimum values of $\mathrm{P} / \mathrm{Py}$, wherein a cross-section local buckling moment capacity coincides with its material strength moment capacity. These values are plotted against the corresponding values of P/Py. Beyond this value of P/Py, a sharp knee portion occurs and gradually decreases.

\section{Non-Compact Beam-Columns.}

The local buckling interaction curves for non-compact beam-columns. These curves correspond to flange slenderness and various web slendernesses. As Mcr /My increases from zero, for each curve a point of tangency to the straight line joining the points $\mathrm{P} / \mathrm{Py}=1.0$ and $\mathrm{Mcr} / \mathrm{My}=1.04$ is approached. At this point, the mode of failure changes from web local buckling to flange local buckling, $t$ h e sloping dashed line, Myc $=$ My (1 - P/Py), represents the strength interaction equation for non-compact beam-columns.

\section{Residual Stresses.}

The interaction curves include the effects of residual stresses. A linearly varying residual stress pattern was assumed, with a maximum compressive stress of $30 \%$ of yield, at the flange tips and maximum tensile stresses at web to flange junctions and web center. For comparison, the interaction curves for plastic design beam-column cross sections with no residual stresses. The rounded knee portions of the curves are due to residual stresses which result in gradual yielding within a cross section, the rounded knee portions change to abrupt transitions in the absence of residual stresses. 


\section{Effects of Strain-Hardening.}

Varying the value of the strain-hardening modulus affects local buckling in the strain-hardening range only. The effect of varying Es is seen to be most significant for low values of for Mcr/Mp greater than about 1.05 in the region where flange buckling governs the behavior.

\section{Columns}

Most thick-walled columns, regardless of whether they buckle elastically or inelastically, fail at loads close to the critical load. The post-buckling curves of perfect members are stable for elastic buckling, and initially stable and then unstable for inelastic buckling. However, each of these curves has a relatively small slope, and consequently there exists neither post-buckling strength for columns that buckle elastically nor imperfection sensitivity in columns that buckle inelastically. By comparison, thin-walled columns may experience local or plate buckling prior to overall failure of the member. If the local buckling occurs within the elastic range, and if the unloaded edges of the elements that make up the column are sufficiently stiff, the load at which failure of the member takes place may be significantly higher than the load at which the individual elements begin to buckle. Thin-walled columns can thus be said to exhibit post-buckling strength. A column stability problem of quite a different nature results when continuously welded railroad tracks are considered. Due to their inability to expand freely with increases in temperature, these members are subject to thermal buckling.

\section{Beams}

Beams that buckle elastically by a combination of twisting and bending about their minor axis, i.e., lateral buckling, have a stable post-buckling curve. If the beam is determinate and of ordinary proportions the post-buckling strength is insignificant and can be neglected. However, indeterminate beams have the ability to redistribute the primary bending moment subsequent to the onset of buckling, and consequently exhibit sizeable post-buckling strength. The different methods of attaching the ends of the column will lead to different likehoods of collapse. These different end conditions have been analyzes and are presented as a set of effective lengths, i.e., by how much and in which direction (longer or shorter) do these different end conditions change the behavior of the column. To put it another way, how long would a pinned-Pinned column have to be to exhibit the same tendency to buckle? Several different end conditions and their corresponding "effective" length.

End conditions

\begin{tabular}{|c|c|c|c|}
\hline End Condition & $\begin{array}{c}\text { Theoretical } \\
\text { Value }\end{array}$ & AISC & $\begin{array}{c}\text { Conservative } \\
\text { value }\end{array}$ \\
\hline Round-Round & Leff $=\mathrm{L}$ & Leff $=\mathrm{L}$ & Leff $=\mathrm{L}$ \\
\hline Pinned-Pinned & Leff $=\mathrm{L}$ & Leff $=\mathrm{L}$ & Leff $=\mathrm{L}$ \\
\hline Fixed-Free & Leff $=2 \mathrm{~L}$ & Leff $=2.1 \mathrm{~L}$ & Leff $=2.4 \mathrm{~L}$ \\
\hline Fixed-Pinned & Leff $=0.707 \mathrm{~L}$ & Leff $=0.80 \mathrm{~L}$ & Leff $=\mathrm{L}$ \\
\hline Fixed-Fixed & Leff $=0.5 \mathrm{~L}$ & Leff $=0.65 \mathrm{~L}$ & Leff $=\mathrm{L}$ \\
\hline
\end{tabular}

Another interesting and important aspect of this analysis is that as beams get shorter (into a region we call intermediate) they exhibit the tendency to fail at loads less than are predicted using the Euler formula. This has lead to the development of a Companion expression (using a parabolic curve fit) to properly account for failures in this intermediate region. This parabolic equation, first suggested by J.B.Johnson now bears his name. The point at which we shift from the Euler formula to the J.B.Johnson formula is usually taken as half the yield strength of the material, i.e. Sy/2. Thus in doing a column buckling analysis, once one has recognized that buckling failure mode exists, into compute the Slenderness ratio of the column and the Slenderness ratio at the dividing line between J.B.Johnson parabola, and Euler and determine which expression is appropriate for calculating critical load.

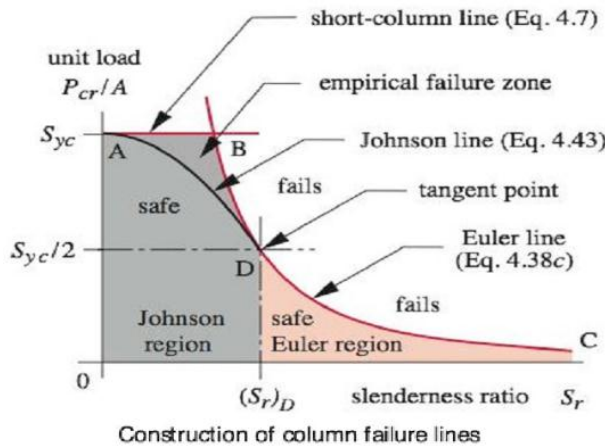

www.iosrjournals.org 


\section{Recent Developments}

Recently researchers have been developing powerful analytical techniques primarily for use with high speed digital computers. The effects of residual stresses were not included and a linear material response was assumed. The method gave good results for flange local buckling but web local buckling could not be predicted accurately. The buckling modes were restricted by the assumption that straight lines across the flanges and normal to the web remain straight during buckling. Although the method is quite general with regard to plate geometry and boundary conditions, it assumes linear elastic response and does not include the effects of residual stresses. A survey of post-buckling behavior of structures is presented. Using simple models, the basic characteristics of the buckling and post-buckling process are outlined. Included are discussions of stable and unstable post-buckling behavior and of the role of imperfections in stability behavior. Reference is made to the behavior of specific structural elements including thick and thin walled columns, beams, plates, cylindrical and spherical shells, and arches.

\section{Scope Of Investigation}

In the investigation presented herein, the analytical technique referred to previously is used to conduct an extensive study of the geometric and material parameters which significantly influence the local buckling behavior of I shapes. The results of this parametric study, carried out separately for columns and beams, are presented in graphical form. As a result of this exhaustive theoretical investigation, several of the existing plate slenderness code requirements are substantiated, while in some cases, suggested modifications to existing code requirements are indicated.

\section{Conclusion}

In this study we are concluded that, before designing of structure it is necessary to know about buckling behavior of beam-column connection and beam, column. For that we know, plastic design section, compact section and non compact section. Experimental as well as analytical procedure takes place for finding out buckling effect in beam and column. This behavior of the analyzed structures signifies that under loaded condition the structure does not become unstable and buckle immediately. Therefore it is important to study buckling behavior of beam, column and beam- column.

\section{References}

[1]. Local Buckling Behavior Of Beam-Columns, By John L. Dawe,1 M. ASCE, and Geoffrey L. Ku, JOURNAL OF STRUCTURE ENG. 1986.112:2447- 2461.lak,2 F. ASCE

[2]. Local Buckling Of W Shape Columns And Beam, (By John L. Dawe1 And Geoffrey L. Kulak/ Members, ASCE) J. STRUCT. ENG. 1984.110:1292-1304.

[3]. Plate Instability Of W Shapes (By John L. Dawe, 1 And Geoffrey L. Kulak, 2 Members, ASCE) J. Struct. Eng. 1984.110:12781291.

[4]. Post-buckling Instability of Steel Beam-columns, (By Masayoshi Nakashima, A. M. ASCE, Takeshi Nakamura/ And Minoru Wakabayashi, M. ASCE) J. Struct. Eng. 1983.109:1414-1430.

[5]. Evaluation on the Post-buckling Residual Strength of H-shaped Steel Column (Hwon-mo Parka, Jae-hyouk Choi ) 1877-7058 (C) 2011 Published by Elsevier

[6]. Local, Distortional,\& Euler Buckling Of Thin Walled Columns (B. W. Schafer,m.Asce) Journal Of Structural Engg./ March 2002

[7]. Locally Buckled Thin-walled Column (By Gale P. Mulligan,1 A. M. ASCE And Teoman Pekoz,2 M. Asce) J. Struct. Eng. 1984.110:2635-2654.

[8]. Local And Overall Buckling In Thin-walled Beams And Columns, (By J. D. Toneff,1 S. F. Stiemer,2 And P. Gstemeder)J. Struct. Eng. 1987.113:769-786.

[9]. Lateral-torsional Buckling Of Thin-walled I-beams,(By Masahiro Kubo1 And Yushi Fukumoto, Member, ASCE) J. Struct. Eng. 1988.114:841-855.

[10]. Post-buckling Behavior, (By Alexander Chajes,1 M. Asce) J. Struct. Eng. 1983.109:2450-2462.

[11]. Dawe, J. L., "Local Buckling of W Shapes Used as Columns, Beams, and Beam-Columns," thesis presented to the University of Alberta, Edmonton,Alberta,Canada in 1980 in partial fulfillment of the requirements for the degree of Doctor of Philosophy.

[12]. Dawe, J. L., and Kulak, G. L., "Plate Instability of W Shapes," Journal of the Structural Division, ASCE, Vol. 110, No. 6, June, 1984, pp. 1278-1291.

[13]. 13) Dawe, J. L., and Kulak, G. L., "Local Buckling of W Shape Columns and Beams," Journal of the Structural Division, ASCE, Vol. 110, No. 6, June, 1984, pp. 1292-1304. 